\title{
Stability Analysis of Interconnected Power Systems Coupled With Market Dynamics
}

Fernando L. Alvarado, Fellow, IEEE, Jianping Meng, Student Member, IEEE, Christopher L. DeMarco, Member, IEEE, and Wellington S. Mota, Member, IEEE

\begin{abstract}
The use of market mechanisms to determine generation dispatch, and the natural tendency to seek improved economic efficiency through rapid market updates, raises a critical issue. As the frequency of market-based dispatch updates increases, there will inevitably be interaction between the dynamics of markets determining the generator dispatch commands, and the physical response of generators and network interconnections. This paper examines questions of stability in such coupled systems by means of numeric tests using various market update models, (including detailed generator/turbine/governor dynamics) for the New England 39 bus test system. The results highlight the nature of potential instabilities and show the interaction modes between physical and market quantities through eigen-analysis. Understanding of potential modes of instability in such coupled systems is crucial both for designing suitable rules for power markets, and for designing physical generator controls that are compatible with market-based dispatch.
\end{abstract}

Index Terms-Dynamic coupling, eigenvalues, market dynamics, power system dynamics.

\section{INTRODUCTION}

$\mathbf{T}$ HE POTENTIAL benefits of near real time competitive markets to determine power production in the electric power industry has long been discussed. A key element for realizing potential benefits from markets is the presence of a robust transmission grid that allows widespread access of power consumers to a range of (often geographically disperse) generators controlled by competitive producers. In general, as networks around the world have become better connected, and the number of potential options available has increased, the pressure for reliance on market mechanism has grown stronger [7]. However, these same conditions (large numbers of widespread generation sources coupled to a long distance transmission network) create a physical system in which the potential for undesirable dynamic response, and even electromechanical instability, is very real.

When designing power exchanges and policies for Independent System Operators (ISO) to manage competitive provision of electric power, it is necessary to consider whether the market operation itself leads to stable equilibrium conditions. At the same time, the physical power system must

Manuscript received February 14, 2000; revised December 27, 2000. This work was supported by Subcontract 35352-6087 from Cornell University, under WO 8333-04 from the Electric Power Research Institute and the US Army Research Office.

F. L. Alvarado, J. Meng and C. L. DeMarco are with the Department of Electrical and Computer Engineering, University of Wisconsin-Madison.

W. S. Mota is with the Department of Electrical Engineering, Federal University of Paraiba, Brazil.

Publisher Item Identifier S 0885-8950(01)09428-7. exhibit acceptable electromechanical response (with stability as a minimum criterion). If market mechanisms are used to determine generator dispatch (with near real-time updates of the dispatch commands) there will exist dynamic coupling between the market update process and the physical response of the generator/network dynamics.

Therefore, under the assumption of market-based dispatch, considering of the stability of the coupled system incorporating both market operation and electromechanical power system dynamics simultaneously is necessary. There has been relatively little prior work in this topic within power systems literature. As rules for market-based dispatch vary throughout the US and around the world, any analytic treatment must make certain assumptions of how physical system conditions and market response interact. Alvarado [2], [3] considered the effect of coupling in one direction, with energy imbalance in the physical system, with or without network congestion, driving the market response. The work by Mota and Alvarado [4] gives the basic modeling for full, two-way dynamic coupling between market dynamics and power system electromechanical response. A discrete time frequency domain formulation is discussed by Bhattacharya in [12].

This paper refines the modeling in [4] and proposes a new frequency error coupling model. Furthermore, the stability of market-only dynamics and coupled system dynamics is examined in system parameter space based on a larger test network.

Before further discussion, it is useful to state the underlying assumptions [2], [3]:

- Marginal production costs are affine linear functions of generated power.

- Marginal benefit functions are negatively sloping affine linear functions of power consumption.

- A generator's power output command is a function of its marginal cost and the market price for power. Demand is also a function of marginal benefit and power price.

- Response of power suppliers and consumers to observed price is represented by continuous dynamics (as opposed to a discrete-time model of periodic price updates). The producer/consumer response characteristics are represented by first order linear differential equations.

- Network-wide power production is not precisely balanced to power consumption at all times; therefore instantaneous energy imbalance and frequency variation result. Energy imbalance leads to the need to control such imbalance to prevent system damage or unwanted relay action.

Section II describes the behavior of power producers and consumers in a market driven environment. Section III presents 
TABLE I

TyPical RANGe FOR POWER MARKET PARAMETERS (POWER IN p.u. With 100 MW BASE)

\begin{tabular}{c|c|c|c|c|c}
\hline & $\lambda$ & $c_{g}$ & $c_{d}$ & $b_{g}, b_{d}$ & $d P_{g} / d t, d \bar{P}_{d} / d t$ \\
\hline Units & $\$ / M W h$ & $\$ / M W^{2} h$ & $\$ / M W^{2} h$ & $\$ / M W h$ & $p . u . / \min (100 \mathrm{MW}$ base $)$ \\
\hline Typical Range & $20 \sim 80$ & $0.004 \sim 0.012$ & $-0.013 \sim-0.003$ & $20 \sim 60$ & $1 / 100 \sim 20 / 100$ \\
\hline
\end{tabular}

the ideal energy imbalance driven market-only dynamic model. Section IV outlines the power system dynamic models. Section V proposes the improved frequency error market-only dynamic model. When the interconnected power system is coupled with market dynamics, the combined system can exhibit the dynamic behavior which is different from that of each subsystem. This is discussed in Sections VI and VII. In order to check the effect of the interaction between the two subsystems, numerical eigenanalysis is used to characterize possible interactions, using the example of the New England 39 bus test system [14]. Variation in key parameters is examined to determine ranges of values that yield stable operation.

The numerical studies suggest that to create a successful power market structure, market designers and regulators cannot ignore the dynamic interaction between the market and the physical, electromechanical dynamics of the power system.

The analysis of this paper applies directly to any market where real time prices are computed and adjusted sufficiently rapidly (e.g., PJM, California, etc.) and where the generation (and possibly the loads) are permitted to respond to the posted prices.

\section{Producer/CONSUMER BeHAVIOR}

By neglecting network losses, ${ }^{1}$ the producer and consumer behavior for $m$ power producers and $n$ consumers in a market driven environment can be approximated by the following simple first order differential equations

$$
\begin{aligned}
& \tau_{g i} \dot{P}_{g i}=-b_{g i}-c_{g i} P_{g i}+\lambda, \quad i=1 \cdots m \\
& \tau_{d j} \dot{P}_{d j}=b_{d j}+c_{d j} P_{d j}-\lambda, \quad j=1 \cdots n
\end{aligned}
$$

where

$$
\begin{array}{ll}
P_{g i} & \text { Power supply of producer } i \\
P_{d j} & \text { Power demand of consumer } j \\
b_{g i}+c_{g i} P_{g i} & \text { Marginal cost of supplier } i \\
b_{d j}+c_{d j} P_{d j} & \text { Marginal benefit of consumer } j \\
\lambda & \text { Price of power. }
\end{array}
$$

The above equations describe the following qualitative behavior: a generator increases its production when price exceeds its marginal production cost. Loads act to increase consumption when marginal benefit exceeds price. Each maximizes its profit or benefit by matching its marginal cost/benefit to market price at equilibrium. First order differential equations allow approximate representation of generator ramp rates and lags in response to power price changes.

\footnotetext{
${ }^{1}$ Consideration of losses in the stability analysis of the paper would require the use of the complete power flow model for the entire network as part of the formulation, or at least the inclusion of penalty factors. Since some markets operate successfully without explicit inclusion of losses, we consider this an unnecessary complication at this time. However, extension of this work to include losses is straightforward and should not affect our conclusion.
}

The units of measure and assumed range for power price $\lambda$, power supply $P_{g}$ and power demand $P_{d}$, power producer cost parameters $c_{g}$ and $b_{g}$, power consumer benefit parameters $c_{d}$ and $b_{d}$, the ramp rate of $P_{g}$ and $P_{d}$ are listed in Table I. In our numerical example to follow, the parameters of $c_{g}, c_{d}, b_{g}, b_{d}$ are chosen to match the above equilibrium equations and the power flow results for the New England 39 bus system based on a hypothetical power price of $\$ 40 / \mathrm{MWh}$. Once the right hand side parameters in the above equations are determined, one may choose producer and consumer response constants $\tau_{g}$ and $\tau_{d}$ by assuming that the ramp rate for generators and consumers lies in the range of $1 \sim 20 \mathrm{MW} / \mathrm{min}$. The specific power producer/ consumer parameters are given in Table V.

\section{ENERGY IMBALANCE MARKET DYNAMICS}

In a synchronous power system, energy imbalance cannot be sustained indefinitely. It must be reduced or driven to zero. In the traditional utility environment in the US, this objective is attained by automatic generation control (AGC) [11]. In a real-time market-driven model, it is reasonable to assume that market mechanisms might fulfill this role. In particular, one may hypothesize that price for power reflects the degree of energy imbalance. That is, an excess of power supplied to the grid depresses the value of the power, and vice versa. This concept was the basis for the proposals by Caramanis et al. [8] and has been recently promoted further by [9], [10]. Variations in the value of power depending real time energy balance is referred as frequency regulation pricing or ACE (Area Control Error) pricing. Under this model, and neglecting network loss effects, we propose the following equations to represent the ideal market-only dynamics for $m$ power suppliers and $n$ power consumers:

$$
\begin{aligned}
\dot{E} & =\sum_{i=1}^{m} P_{g i}-\sum_{j=1}^{n} P_{d j} \\
\tau_{\lambda} \dot{\lambda} & =-k_{E} E-\lambda
\end{aligned}
$$

where

$E \quad$ System energy imbalance;

$\tau_{\lambda} \quad$ Power price response rate constant;

$k_{E} \quad$ Market stabilizer gain for energy imbalance.

The parameters $\tau_{\lambda}$ and $k_{E}$ depend on the design of the power market. The result of an excess of energy is assumed to be a reduction of power price derivative, which changes according to the ratio $k_{E} / \tau_{\lambda}$. This ratio dictates the sensitivity of price to system energy imbalance $E$. Although power price feedback in (4) tends to stabilize the market, it is optional. Its presence depends on the market design. Without price feedback, the change of price is dependent only on energy imbalance, and steady state energy imbalance is driven to zero. With price feedback, the change of price depends not only on energy imbalance but also 


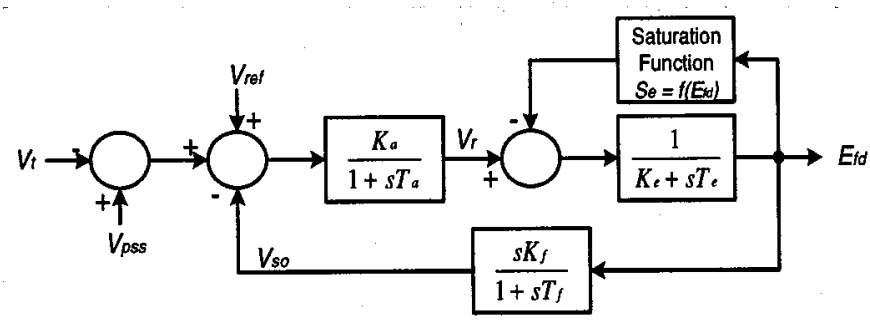

Fig. 1. IEEE TYPE 1 exciter system.

on the current price, and there remains a steady state error in energy imbalance. In this paper we use (4) which includes price feedback.

\section{POWER SYSTEM DYNAMICS}

The synchronous machines, exciters and voltage regulators, turbines and governors, are represented by well-established, textbook models used for electromechanical stability studies [5], [6].

\section{A. Synchronous Machine Model}

Synchronous machines are modeled by either a 3rd order flux decay model or a 4th order two axis model depending on the specification for each synchronous machine. Only the 4th order two axis synchronous model is presented here; readers are referred to [5], for other synchronous machine models.

State Equations

$$
\begin{aligned}
T_{q 0}^{\prime} \dot{E}_{d}^{\prime} & =\left(x_{q}-x_{q}^{\prime}\right) I_{q}-E_{d}^{\prime} \\
T_{d 0}^{\prime} \dot{E}_{q}^{\prime} & =E_{f d}-\left(x_{d}-x_{d}^{\prime}\right) I_{d}-E_{q}^{\prime} \\
\dot{\delta} & =\omega_{s} \omega \\
2 H \dot{\omega} & =P_{m}-\left[E_{q}^{\prime} I_{q}+E_{d}^{\prime} I_{d}-\left(x_{d}^{\prime}-x_{q}^{\prime}\right) I_{d} I_{q}\right]-D \omega
\end{aligned}
$$

where $\delta$ is the rotor angle relative to the synchronous reference angle. The quantity $\omega$ is the per unit rotor speed deviation relative to the synchronous reference.

Stator Algebraic Equations

$$
\begin{aligned}
& 0=E_{d}^{\prime}-V_{d}-r_{a} I_{d}+x_{q}^{\prime} I_{q} \\
& 0=E_{q}^{\prime}-V_{q}-x_{d}^{\prime} I_{d}-r_{a} I_{q}
\end{aligned}
$$

where

$$
\begin{aligned}
& V_{d}=\operatorname{Re}\left(\vec{V}_{g} e^{j(\pi / 2-\delta)}\right)=V_{g} \sin (\delta-\theta) \\
& V_{q}=\operatorname{Im}\left(\vec{V}_{g} e^{j(\pi / 2-\delta)}\right)=V_{g} \cos (\delta-\theta)
\end{aligned}
$$

$\vec{V}_{g}$ is the generator terminal voltage phasor, $V_{g}$ is the amplitude of $\vec{V}_{g}, \theta$ is the phase angle of $\vec{V}_{g}$.

\section{B. Exciter and Automatic Voltage Regulator (AVR) Model}

Only IEEE type 1 exciter and AVR model is considered. The block diagram of this model is shown in Fig. 1. The PSS is assumed to be inactive. The exciter saturation function is $S_{e}\left(E_{f d}\right)=0.0039 e^{1.555 E_{f d}}[6]$.

$$
T_{e} \dot{E}_{f d}=-\left[K_{e}+S_{e}\left(E_{f d}\right)\right] E_{f d}+V_{r}
$$

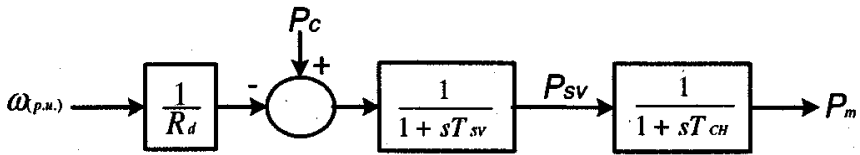

Fig. 2. Simplified speed-governor system.

$$
\begin{aligned}
T_{a} \dot{V}_{r} & =-V_{r}-K_{a} V_{s o}+K_{a}\left(V_{r e f}-V_{t}\right) \\
T_{f} \dot{V}_{s o} & =-V_{s o}-K_{f} \frac{K_{e}+S_{e}\left(E_{f d}\right)}{T_{e}} E_{f d}+K_{f} \frac{V_{r}}{T_{e}} .
\end{aligned}
$$

\section{Turbine and Speed Governor Model}

The block diagram of the turbine and speed governor model is shown in Fig. 2.

$$
\begin{gathered}
T_{C H} \dot{P}_{m}=-P_{m}+P_{S V} \\
T_{S V} \dot{P}_{S V}=-P_{S V}+P_{c}-\frac{\omega}{R_{d}} .
\end{gathered}
$$

In Fig. 2, $\omega$ is the per unit rotor speed deviation relative to synchronous speed, $P_{c}$ is the power command, and $P_{m}$ is the generator mechanical power.

If the market dynamics are considered, the power command $P_{c}$ becomes the input point for coupling; it is set equal to the output $P_{g}$ from the market dynamics model.

\section{Power Flow and Load Representation}

At generator buses the power balance equations are:

$$
\vec{S}_{t i}-P_{L i}-j Q_{L i}-\vec{S}_{g i}(\theta, V)=0
$$

where

$\vec{S}_{t i}$

$P_{L i}+j Q_{L i} \quad$ terminal complex load at generator bus $i$;

$\vec{S}_{g i}$

complex power injection at generator bus $i$, which is the function of bus voltages;

$V \quad$ bus voltage magnitude vector;

$\vec{I}_{g i}^{*} \quad$ conjugate of the generator current at generator bus $i$.

The above equations can be rewritten as:

$V_{i} e^{j \theta_{i}}\left(I_{d i}-j I_{q i}\right) e^{-j\left(\delta_{i}-\pi / 2\right)}-P_{L i}-j Q_{L i}-\vec{S}_{g i}(\theta, V)=0$.

At load buses the power balance equations are:

$$
P_{L j}+j Q_{L j}+\vec{S}_{L j}(\theta, V)=0
$$

where $P_{L j}+j Q_{L j}$, is the power consumption at load bus $j \cdot \vec{S}_{L j}$ is the complex power injection at load bus $j$. In power flow computation, loads are modeled as constant power. In power system dynamic analysis, loads are modeled as constant admittances. When market dynamics are considered, loads at nonconsumer 


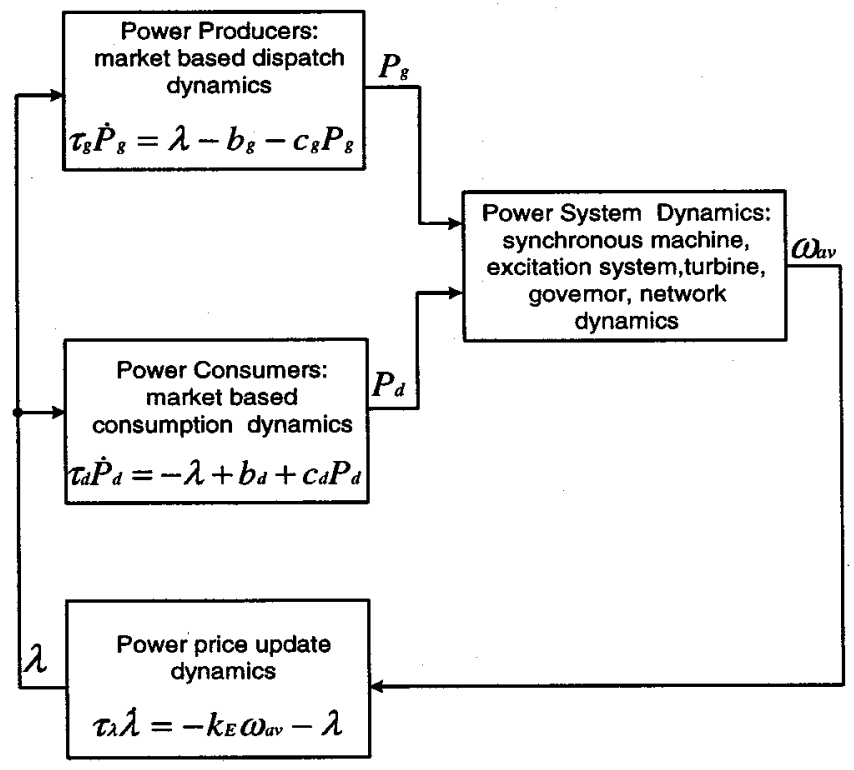

Fig. 3. Coupled market/power system dynamic model.

buses (e.g., loads in power plants) are represented as admittances and absorbed by the bus admittance matrix. At a power consumer bus $j$, the real power balance equation is modified as:

$$
P_{d j}+\operatorname{Re}\left(\vec{S}_{L j}(\theta, V)\right)=0
$$

\section{RELATION OF FREQUENCY ERROR TO ENERGY IMBALANCE}

The preceding sections present the ideal market-only dynamic model in terms of the system energy imbalance $E$. In real systems such abstract energy imbalance is impossible to measure. When the coupling between power system dynamics and market dynamics is considered, a way must be found to measure such energy imbalance. By analyzing the generator rotor acceleration equation (8) and ignoring network losses, the power demand is approximated by the sum of the square bracketed terms in (8). Since the damping term usually is small, the sum of all right hand sides of (8) closely approximates system power imbalance, and thus integrates to energy imbalance. Thus the weighted sum of frequency errors $\omega_{a v}=\sum_{i}^{m}$ $2 H_{i} \omega_{i}$ is a good approximation to system energy imbalance. The frequency error market-only dynamic model is described by the following first order differential equations,

$$
\begin{aligned}
\tau_{g i} \dot{P}_{g i} & =-b_{g i}-c_{g i} P_{g i}+\lambda, \quad i=1 \cdots m \\
\tau_{d j} \dot{P}_{d j} & =b_{d j}+c_{d j} P_{d j}-\lambda, \quad j=1 \cdots n \\
\tau_{\lambda} \dot{\lambda} & =-k_{E} \omega_{a v}-\lambda
\end{aligned}
$$

where $\omega_{a v}=\sum_{i}^{m} 2 H_{i} \omega_{i}$ is the average frequency deviation.

For the coupled market/power system, $P_{g}$ is set to equal to the governor input $P_{c}$. The coupled market/power dynamic model can be best understood from the diagram of Fig. 3 .

\section{MARKET/POWER SYSTEM LINEARIZED MODEL}

The combined market/power system yields a set of differential/algebraic equations. The linearized version of the combined market and power system differential and algebraic equations has a structure that may be summarized in matrix form:

$$
\left[\begin{array}{c}
\frac{\Delta \dot{P}_{g}}{\Delta \underline{\dot{P}}_{d}} \\
\frac{\Delta \dot{\lambda}}{\Delta \underline{\dot{X}}_{g e n}} \\
\hline \underline{0} \\
\underline{0} \\
\underline{0} \\
\underline{0} \\
\underline{0}
\end{array}\right]=\left[\begin{array}{l|c|c}
\mathbf{J}_{m m} & \mathbf{J}_{m g} & \mathbf{J}_{m a} \\
\hline \mathbf{J}_{g m} & \mathbf{J}_{g g} & \mathbf{J}_{g a} \\
\hline \mathbf{J}_{a m} & \mathbf{J}_{a g} & \mathbf{J}_{a a}
\end{array}\right]\left[\begin{array}{c}
\Delta \underline{P}_{g} \\
\Delta \underline{P} d \\
\frac{\Delta \lambda}{\Delta \underline{X}_{g e n}} \\
\hline \Delta \underline{I}_{d} \\
\Delta \underline{I}_{q} \\
\Delta \underline{\theta}_{g} \\
\Delta \underline{\theta}_{l} \\
\Delta \underline{V}_{g} \\
\Delta \underline{V}_{l}
\end{array}\right]
$$

where

$\mathbf{J}_{m m}$ Jacobian of power market state equations w.r.t. power market state variables;

$\mathbf{J}_{g m}$ Jacobian of generator state equations w.r.t. power market state variables;

$\mathbf{J}_{a m}$ Jacobian of power system algebraic equations w.r.t. power market state variables;

$\mathbf{J}_{g g}$ Jacobian of generator state equations w.r.t. generator state variables;

$\mathbf{J}_{g a}$ Jacobian of generator state equations w.r.t. power system algebraic variables;

$\mathbf{J}_{a g}$ Jacobian of power system algebraic equations w.r.t. generator state variables;

$\mathbf{J}_{a a}$ Jacobian of power system algebraic equations w.r.t. power system algebraic variables;

$\mathbf{J}_{m a}$ Jacobian of power market state equations w.r.t. power system algebraic variables;

$\mathbf{J}_{a m}$ Jacobian of power system algebraic equations w.r.t. power market state variables.

Using the Schur complement formula, ${ }^{2}$ the eigenvalues of the coupled dynamic system can be computed from the matrix of (25).

\section{NeW ENGLAND 39 Bus SySTEM EXAMPLE}

The effect of the interaction between the market and the power system is illustrated using the classical New England 39 bus system. All the synchronous machines are modeled as two axis 4th model except bus 1 which is treated as a 3 rd order flux decay model. All generator exciters and voltage regulators are IEEE type 1 . The turbines and speed governors for all the generators are modeled using the aforementioned 2nd order model. The original system data can be found in [1], [14].

\section{A. Stability of the Power System}

Using the modeling discussed in the preceding sections, the computation of eigenvalues for system dynamics is straightforward. The eigenvalue plot for this stable power system is shown in Fig. 4. There is a 0 eigenvalue. It comes from keeping all generator rotor angles $\underline{\delta}$ as state variables. If synchronous machines damping is ignored, a second zero eigenvalue appears [6].

\footnotetext{
${ }^{2} \mathrm{An}$ augmented solution is also possible [13]
} 


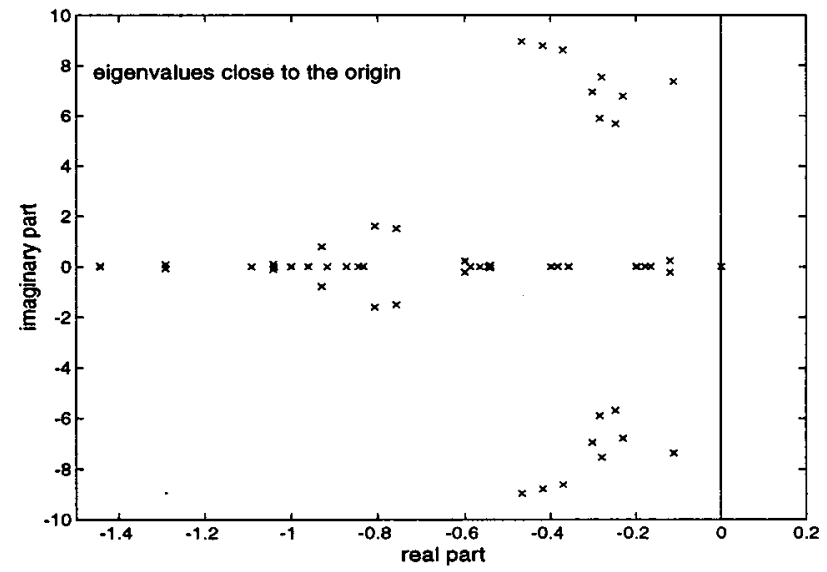

Fig. 4. Eigenvalue plot of stable New England system.

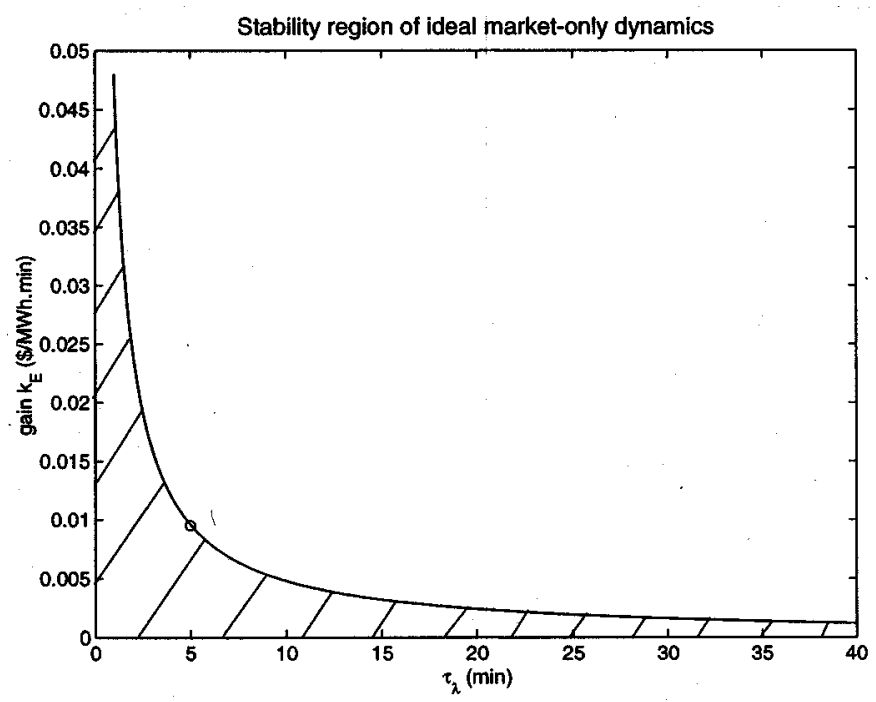

Fig. 5. Stability region of the ideal market-only dynamics w.r.t. $k_{E}$ and $\tau_{\lambda}$ for the New England system.

\section{B. Stability of Ideal Market-Only Dynamics}

This section analyzes the stability of the ideal market-only dynamic model presented in Sections II and III. If the market dynamics are not coupled with the electric power system, the computation of the eigenvalues associated with the continuous market dynamics model is trivial. The market parameters are given in the Appendix.

Assume the market parameters associated with power producers and consumers are fixed. The stability region for the ideal market-only dynamics with respect to market parameters $k_{E}$ and $\tau_{\lambda}$ is the shaded area in Fig. 5. For a specific pair of $\tau_{\lambda}=5 \mathrm{~min}$ and $k_{E}=0.0095 \$ \mathrm{MWh} \cdot \mathrm{min}$ (see Fig. 5), there is a pair of critically stable modes $-0.0006 \pm 0.0616 i .^{3}$ The computation of the corresponding normalized participation factors shows that these two modes are mainly associated with system energy imbalance $E$ and power price $\lambda$ with the corresponding normalized participation factors 1.00 and 0.30 , respectively.

${ }^{3}$ Markets in general tend to exhibit stable but oscillatory behavior with some poorly damped modes common. Power systems are generally designed to have only well damped modes. These different features of markets and power systems must be taken into consideration when interpreting results from this work.

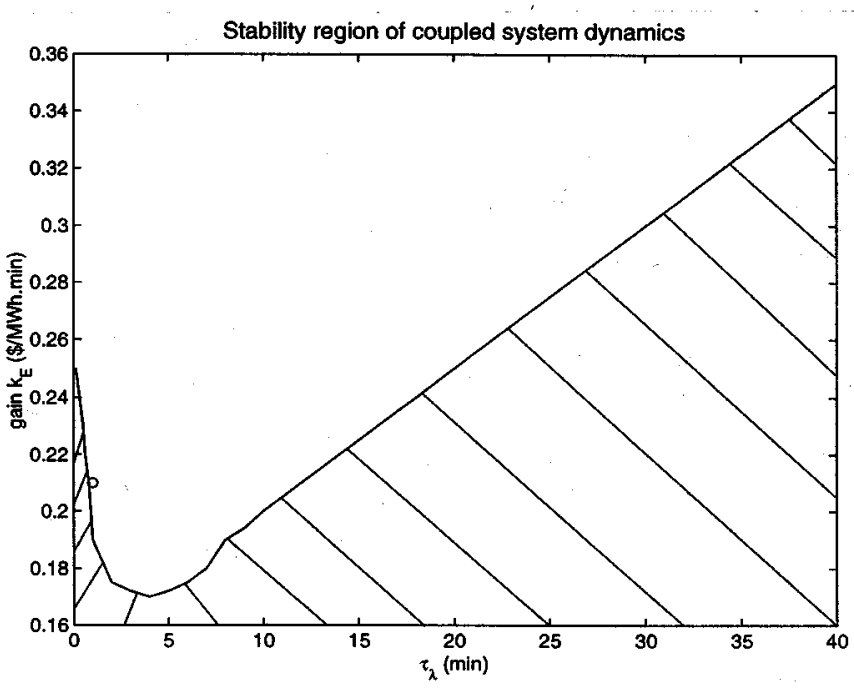

Fig. 6. Stability region of the coupled system dynamics w.r.t. $k_{E}$ and $\tau_{\lambda}$ for New England system.

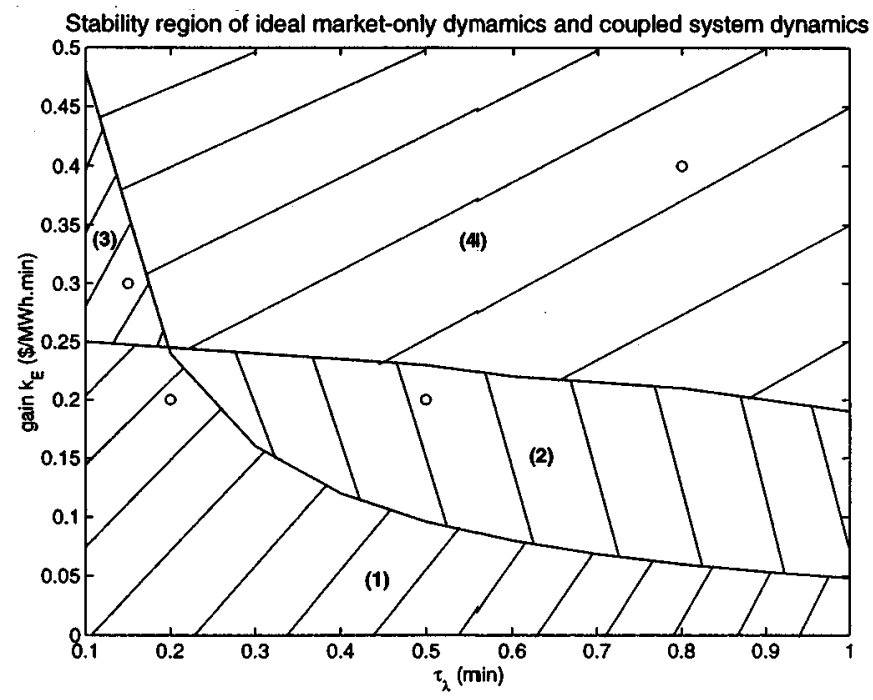

Fig. 7. Stability regions of the ideal market-only and coupled system dynamics w.r.t. $k_{E}$ and $\tau_{\lambda}$ for New England system.

\section{Stability of Interconnected System Coupled With Frequency Error Market Dynamics}

Consider next the stability of the coupled system. In order to examine the mechanism of power market design, the frequency error market dynamic model, described in Section V, is used here. Using the formulation derived in Section VI, the eigenvalues of the coupled system can be easily calculated. The stability region with respect to the market parameters $k_{E}$ and $\tau_{\lambda}$ is shown as the shaded area in Fig. 6. For the pair of $\tau_{\lambda}=1 \mathrm{~min}$, $k_{E}=0.21 \$ / \mathrm{MWh} \cdot \mathrm{min}$, (see Fig. 6), there is one pair of critically unstable modes $0.0019 \pm 0.2533 i$. The normalized participation factors associated with power supply $P_{g}$ of power producers, power price $\lambda$, machine rotor angles $\delta$, the machine rotor speed deviation $\omega$, and the turbine mechanical power output $P_{m}$ are shown in Table II. For this specific pair of unstable modes, the states of the swing dynamics of the last machine have more 
TABLE II

Selected Normalized Participation Factors Associated With the UnStable Modes $(0.0019 \pm 0.2533 i)$ For the Coupled System $\left(\tau_{\lambda}=1 \mathrm{~min}, k_{E}=0.21 \$ / \mathrm{MWh} \cdot \mathrm{min}\right.$, the PARTicipation FACTOR AsSOCiated With PRICE $\lambda$ IS 0.74$)$

\begin{tabular}{c|cccccccccc}
\hline Bus \# & 30 & 31 & 32 & 33 & 34 & 35 & 36 & 37 & 38 & 39 \\
\hline$P_{g}$ & 0.20 & 0.20 & 0.28 & 0.20 & 0.27 & 0.19 & 0.23 & 0.20 & 0.22 & 0.21 \\
$\delta$ & 0.089 & 0.044 & 0.068 & 0.058 & 0.082 & 0.0045 & 0.011 & 0.073 & 0.14 & 0.45 \\
$\omega$ & 0.42 & 0.29 & 0.35 & 0.27 & 0.25 & 0.31 & 0.25 & 0.23 & 0.32 & 1.00 \\
$P_{m}$ & 0.22 & 0.20 & 0.26 & 0.20 & 0.26 & 0.19 & 0.24 & 0.20 & 0.23 & 0.22 \\
\hline
\end{tabular}

TABLE III

Selected Normalized Participation Factors Associated With the Sample Point in Region (3) for the Coupled System $\left(\tau_{\lambda}=0.15 \mathrm{~min}, k_{E}=0.3 \$ / \mathrm{MWh} \cdot \mathrm{min}\right.$, THE PARTICIPATION FACTOR ASSOCIATED With PRICE $\lambda$ IS 0.13$)$

\begin{tabular}{c|cccccccccc}
\hline Bus \# & 30 & 31 & 32 & 33 & 34 & 35 & 36 & 37 & 38 & 39 \\
\hline$P_{g}$ & 0.20 & 0.19 & 0.27 & 0.19 & 0.26 & 0.18 & 0.22 & 0.19 & 0.22 & 0.20 \\
$\delta$ & 0.078 & 0.037 & 0.054 & 0.049 & 0.069 & 0.0078 & 0.087 & 0.059 & 0.12 & 0.36 \\
$\omega$ & 0.42 & 0.29 & 0.35 & 0.27 & 0.25 & 0.31 & 0.25 & 0.23 & 0.32 & 1.00 \\
$P_{m}$ & 0.22 & 0.20 & 0.27 & 0.20 & 0.27 & 0.19 & 0.24 & 0.20 & 0.23 & 0.22 \\
\hline
\end{tabular}

TABLE IV

Selected Normalized Participation Factors Associated With the Sample Point in Region (4) for the Coupled System $\left(\tau_{\lambda}=0.8 \mathrm{~min}, k_{E}=0.4 \$ / \mathrm{MWh} \cdot \mathrm{min}\right.$, the PARTICIPATION FACTOR ASSOCIATED With PRICE $\lambda$ IS 0.76$)$

\begin{tabular}{c|cccccccccc}
\hline Bus \# & 30 & 31 & 32 & 33 & 34 & 35 & 36 & 37 & 38 & 39 \\
\hline$P_{g}$ & 0.21 & 0.20 & 0.28 & 0.20 & 0.27 & 0.19 & 0.23 & 0.20 & 0.23 & 0.21 \\
$\delta$ & 0.075 & 0.037 & 0.050 & 0.048 & 0.061 & 0.0084 & 0.079 & 0.056 & 0.11 & 0.33 \\
$\omega$ & 0.42 & 0.29 & 0.36 & 0.27 & 0.25 & 0.31 & 0.25 & 0.23 & 0.32 & 1.00 \\
$P_{m}$ & 0.20 & 0.19 & 0.26 & 0.19 & 0.25 & 0.18 & 0.22 & 0.19 & 0.21 & 0.20 \\
\hline
\end{tabular}

participation due to its relatively large inertia and large contribution to system energy imbalance. Putting the above two plots together on scaled coordinate axes leads to a plot of stability regions for both ideal market-only dynamics and coupled system dynamics. This plot is shown in Fig. 7.

There are four regions (shaded areas) in this plot. Region (1) and (3) are the stability regions for ideal market-only dynamics. Region (1) and (2) are the stability regions for coupled system dynamics. In region (1) both of the market-only system and coupled system are stable. In region (2) the market-only system is unstable, yet the coupled system is stable. In region (3) the market-only system is stable, but the coupled system is unstable. In region (4), both of the systems are unstable. From Fig. 7 it is clear that for the different combination of market parameters $\left(\tau_{\lambda}, k_{E}\right)$, one may have very different stability results. Hence, when designing power market policies, both of market system and electric power system must be considered to ensure reliable and secure operation.

To examine the nature of potential instabilities, consider one sample point, $\left(\tau_{\lambda}, k_{E}\right)$ pair, in each region. These four points are shown in Fig. 7.

- For $(0.2,0.2)$ in region (1), both of the ideal market-only system and the coupled system are stable.

- For $(0.5,0.2)$ in region (2), the coupled system is stable, but the ideal market-only system is unstable with one pair of unstable modes $0.0112 \pm 0.2961 i$, mainly associated with system energy imbalance and power price with the corresponding normalized participation factors 1.00 and 0.15 .
- For $(0.15,0.3)$ in region (3), the ideal market-only dynamics are stable, yet the coupled system is unstable with only pair of unstable eigenvalues $0.0089 \pm 0.2932 i$. Some corresponding normalized participation factors associated with this pair of unstable modes are listed in Table III.

- For $(0.8,0.4)$ in region $(4)$, both of the market-only system and the coupled system are unstable. The only one pair of unstable modes for ideal market-only dynamics is $0.0501 \pm 0.4014 i$, which are mainly associated with system energy imbalance and power price with the corresponding normalized participation factors 1.00 and 0.30 , respectively. The unstable mode for the coupled system is $0.0379 \pm 0.3025 i$. Some normalized participation factors associated with these two eigenvalues are listed in Table IV.

\section{CONCLUSION}

This paper begins from a simple, intuitive dynamic model for consumer/producer response and price setting in an electric power market. Energy imbalance (the integral of power supply and demand mismatch) is hypothesized as the key driving term for updating price. A weighted average frequency is used as an approximation to energy imbalance. A coupled dynamics model that encompasses consumer/supplier response, market price update, and the physical power/system dynamics is considered.

The key contribution of the paper is to examine the dynamic impact of market design parameters (such as the sensitivity of price to energy imbalance, or to its surrogate, average frequency 
error). In the market-only dynamic model, the system appears able to tolerate high sensitivity of market price to energy imbalance. In the more accurate coupled model that includes physical dynamics, this sensitivity behaves as a feedback gain, and its value must be much smaller in order to maintain stability.

The implications of this result are significant: those designing the power exchange policies and rules for ISOs for deregulated power market must accommodate to the dynamic needs of the system, and those designing system electromechanical controls must take into consideration the conditions that will be imposed on the power system by operation in a market-driven environment.

\section{ACKNOWLEDGEMENT/DISCLOSURE}

This report has not been reviewed to determine whether it contains patentable subject material, nor has the accuracy of its information or conclusions been evaluated by EPRI.

\section{APPENDIX}

TABLE V

Market Data For the New ENGLAND System

\begin{tabular}{c|c|c|c}
\hline Bus \# & $\tau_{g}(s)$ & $c_{g}\left(\$ / M W^{2} h\right)$ & $b_{g}(\$ / M W h)$ \\
\hline 30 & 35 & 0.8 & 30.00 \\
31 & 30 & 0.7 & 35.99 \\
32 & 25 & 0.7 & 35.45 \\
33 & 30 & 0.8 & 34.94 \\
34 & 25 & 0.8 & 35.94 \\
35 & 30 & 0.8 & 34.80 \\
36 & 30 & 0.8 & 34.40 \\
37 & 30 & 0.8 & 35.68 \\
38 & 30 & 0.6 & 33.36 \\
39 & 35 & $c_{d}\left(\$ / M W^{2} h\right)$ & $b_{d}(\$ / M W h)$ \\
\hline \hline Bus \# & $\tau_{d}(s)$ & -0.8 & 42.58 \\
3 & 150 & -0.7 & 43.50 \\
4 & 150 & -0.6 & 41.40 \\
7 & 150 & -0.6 & 43.13 \\
8 & 155 & -0.8 & 40.07 \\
12 & 150 & -0.8 & 42.56 \\
15 & 150 & -0.7 & 42.31 \\
16 & 155 & -0.6 & 40.95 \\
18 & 150 & -0.8 & 45.44 \\
20 & 155 & -0.7 & 41.92 \\
21 & 150 & -0.7 & 41.73 \\
23 & 150 & -0.6 & 41.85 \\
24 & 155 & -0.6 & 41.34 \\
25 & 155 & -0.7 & 40.97 \\
26 & 150 & -0.7 & 41.97 \\
27 & 150 & -0.8 & 41.65 \\
28 & 150 & -0.7 & 41.98 \\
\hline 29 & 160 & & \\
\hline \hline$\tau_{\lambda}($ min $)$ & $k(\$ / M W h \cdot m i n)$ & & \\
\hline 0.2 & 0.01 & & \\
\hline
\end{tabular}

\section{REFERENCES}

[1] "PacDyn User's Manual—Small signal analysis and control," CEPEL, Rio de Janeiro, Brazil, V4.0-APR/96.

[2] F. L. Alvarado, "The dynamics of power system market," University of Wisconsin-Madison, Pserc Research Report 97-01.

[3] — , "The stability of power system markets," IEEE Trans. Power Systems, vol. 14, no. 2, pp. 509-511, May 1999.

[4] W. S. Mota and F. L. Alvarado, "Eigenvalues calculation of interconnected power system with market dynamics," Pserc Research Report, University of Wisconsin-Madison, 1997.
[5] P. Kundur, Power System Stability and Control: McGraw-Hill, 1993.

[6] P. W. Sauer and M. A. Pai, Power System Dynamics and Stability: Prentice-Hall, 1998.

[7] H.-P. Chao and S. Peck, "A market mechanism for electric power transmission," Journal of Regulatory Economics, vol. 10, pp. 25-59, 1996.

[8] M. C. Caramanis, R. E. Bohn, and F. C. Scweppe, "Optimal pricing in electrical networks over space and time," in Rand Journal of Economics: The University of Wisconsin-Madison, 1984, vol. 15, no. 3, pp. 360-376.

[9] E. Hirst and B. Kirby, "Ancillary-serive details: Regulation, load following, and generation response," ORNL, Oak Ridge, TN, Technical Report ornl/con-433, June 1996.

[10] — , "Ancillary-service details: Dynamic scheduling," Oak Ridge National Laboratory, Oak Ridge, TN, Technical Report ornl/con-438, Jan. 1997.

[11] N. Jaleeli, L. S. Van Slyck, D. N. Ewart, L. H. Fink, and A. G. Hoffmann, "Understanding automatic generation control," IEEE Trans. Power Systems, vol. 7, no. 3, pp. 1106-1122, Aug. 1992.

[12] K. Bhattacharya, "Real-time adaptive pricing for load frequency control of an interconnected power system," International Journal of Power and Energy Systems, vol. 18, no. 2, pp. 102-109, 1998.

[13] N. Martins, "Efficient eigenvalue and frequency response methods applied to power system small-signal stability studies," IEEE Trans. Power Systems, vol. 1, no. 1, pp. 217-226, Feb. 1986.

[14] R. T. Byerly, D. E. Sherman, and R. J. Bennon, "Frequency domain analysis of low-frequency oscillations in large electric power systems," EPRI, 3412 Hillview Avenue, Palo Alto, CA 94304, EPRI EL-726, Project 744-1, Interim Report, 1978.

Fernando L. Alvarado (F'93) is a Professor of Electrical and Computer Engineering at the University of Wisconsin-Madison and a consultant to Christensen Associates. He received the Ph.D. degree from the University of Michigan in 1972. He has numerous publications on numerical methods and computer techniques for power systems application as well as topics on power systems economics analysis. He is Fellow of IEEE.

Jianping Meng (S'99) received the B.S. degree and two M.S. degrees all in electrical engineering from Tsinghua University, North China Institute of Electric Power, and University of Wisconsin-Madison in 1991, 1994, and 1997, respectively. From 1994 to 1995, he was with Sichuan Electric Power Control Center, China, served as a system operator and later an economic dispatch engineer. $\mathrm{He}$ is finishing his Ph.D. degree at the Department of Electrical and Computer Engineering in the University of Wisconsin-Madison.

Christopher L. DeMarco (M'85) received the B.S. degree from Massachusetts Institute of Technology in 1980 and the M.S. and Ph.D. degrees from University of California-Berkeley in 1982 and 1985, all in electrical engineering. He is currently a professor with the Department of Electrical and Computer engineering at the University of Wisconsin-Madison.

Wellington. S. Mota (M'74) was born in Paraiba state, Brazil, on May 12, 1946. $\mathrm{He}$ received the B.Sc. and M.Sc. degrees in electrical engineering from UFPB (Federal University of Paraiba) Brazil in 1970 and 1972, respectively. He received the Ph.D. degree in electrical engineering from Waterloo, University of Waterloo, Canada in 1981. Since 1971, he has been with the Department of Electrical Engineering, UFPB, where currently is a Senior Professor. From 1973 to 1977, he worked at the Sao Francisco River Hydro (CHESF) in power system planning. His research interests include power system dynamics and control, synchronous machine models and market dynamics. 